\title{
Myokine Regulation as Marker of Sarcopenia in Elderly
}

\author{
I Gusti Putu Suka Aryana, Anak Agung Ayu Ratih Hapsari, Raden Ayu Tuty Kuswardhani \\ Geriatric Division, Internal Medicine Department, Faculty of Medicine, Udayana University, Sanglah Teaching Hospital, \\ Denpasar, Indonesia
}

The elderly population will increase as well as increasing life expectancy. Health problems in elderly will be more complex and need a comprehensive management. One of the problems that arise from the aging process is sarcopenia. Sarcopenia is a decreasing in muscle mass and muscle strength or muscle function caused by multifactorial not only due to aging process, but also nutrition, immobilization, genetics and others risk factors. Muscle is an endogen organ that produces various proteins that can affect the health system. This protein is referred to as myokine. Myokine is anti-inflammation cytokine and peptide produced by striated muscles. Physical activity results in myokine secretion that can reduce inflammation due to a sedentary lifestyle. Inflammation can lead to worsening sarcopenia and fat accumulation in striated muscles, thus reducing muscle mass, muscle strength and causing physical inactivity. The most of this type myokine have antiinflammation effect have work as autocrine, paracrine and endocrine. Chronic inflammation is a contributor that plays a role in the pathophysiology of various diseases including sarcopenia, it will protected by myokine. Myokine can affect the metabolism of glucose, fatty acids, angiogenesis, myogenesis, neurogenesis, and can explain the relationship between muscle, liver, fat, tissue and brain. Some knewn myokines include interleukin (IL)-6, IL-8, IL-5, brain-derived neurotrophic factor (BDNF), fibroblast growth factor 21 (FGF-21), leukemia Inhibitory factor (LIF), irisin and secreted protein acidic and rich in cysteine (SPARC). Physical exercise can induce myokine secretion from striated muscle to circulation. Through these mechanisms, myokine is expected to improve metabolism of glucose, fat and protein muscle, liver, fat, tissue, brain and reduce the incidence some comorbidity especially sarcopenia. Finally, it's will be decreasing of disability, morbidity and mortality rate in elderly.

Keywords: myokine, sarcopenia, elderly

\section{Introduction}

The geriatric population is increasing every year. Based on World Health Organization (WHO) data of 2013, in 1950 there was only 202 million geriatric population, increasing 4 fold in 2013 to 841 million inhabitants. This increase is dominated in developing countries where the geriatric population reaches 554 million in 2013 which also increased fivefold from 108 million in 1950. In the geriatric population, there is a decrease in the quality and quantity of striated muscles called sarcopenia which is marked by the loss muscle mass, qualities and functional

Date of submission: March 29, 2018

Last Revised: June 5, 2018

Accepted for publication: June 6, 2018

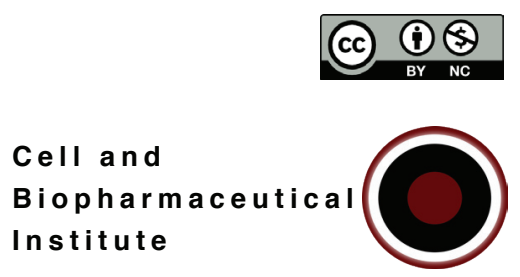

Corresponding Author:

I Gusti Putu Suka Aryana

Geriatric Division, Internal Medicine Department, Faculty of Medicine

Udayana University, Sanglah Teaching Hospital

Jl. Diponogogo, Denpasar, Bali 80113, Indonesia

E-mail: suka_aryana@unud.ac.id 
skeletal muscles progress associated with aging. Sarcopenia will cause physical inactivity, and physical inactivity will lead to increased proinflammatory markers resulting in a vicious cycle of sarcopenia, visceral fat accumulation, cardiovascular disease, type II diabetes, malignancy, dementia and depression called diseasome of physical inactivity. ${ }^{1}$ Sarcopenia also causes a decrease in muscle strength which is one of the causes of frailty that would cause a person to become susceptible to stress and increase the incidence of hospital admission, disability, or even death. $^{2}$

Recent developments prove the striated muscle produces various cytokines and peptides known as a myokine. Some known myokines include interleukin (IL)6, IL-8, IL-5, brain-derived neurotrophic factor (BDNF), fibroblast growth factor 21 (FGF- 21), leukemia inhibitory factor (LIF), irisin and secreted protein acidic and rich in cysteine (SPARC). ${ }^{3}$ Myokine can affect the metabolism of glucose and fat, even related to chronic degenerative diseases. Understanding of the striated muscles as endocrine organs opens our insight into the importance of physical activity in disease prevention, especially in the geriatric population.

\section{Physiology of Striated Muscles}

The striated muscle in our body works voluntarily or is influenced by consciousness. The striated muscle is composed of myofibrils which combine into muscle fibers. The muscle fibers will combine to form the fascism. The combination of fascicles will form the muscle. The striated muscle cells are cylindrical and have many nuclei. Contraction and relaxation begin when the muscles receive stimulation from the nervous system. The neuromuscular junction is the region between the nerve and the muscle in which the neurotransmitter signal is transmitted. The muscle fibers are composed of myofibrils having one contraction unit called sarcomere. Sarcomere consists of two filaments that have thick and thin cross section. Myosin thick filaments attached to the M-line located in the middle of the sarcomere. Whereas the thin filament actin attaches Z-line which is on the edge of the sarcoma. Because actin attached to the Z-line at the edge of the sarcomere actin to myosin attachment to the process of contraction will cause the sarcomere shortens.

The contraction process is expanded when adenosine triphosphate (ATP) in myosin is broken down into adenosine diphosphate (ADP) and phosphate, causing myosin to become erect and attached to actin. Myosin will remain attached to the actin until the new ATP molecule attaches to give the muscle time to relax. Actin consists of two molecules namely troponin and tropomyosin. When muscles relax, tropomyosin blocks actin-attaching sites in myosin. When calcium $\left(\mathrm{Ca}^{2+}\right)$ ions in muscle and ATP are available, $\mathrm{Ca}^{2+}$ will stick to troponin and shift tropomyosin. This causes no inhibitors between actin and myosin, so actin can stick to myosin. $\mathrm{Ca}^{2+}$ is stored in the sarcoplasmic reticulum. The acetylcholine neurotransmitter will be released from the neuron to the neuromuscular junction causing impulse propagation in the $\mathrm{T}$ tubule in the muscle and causing the release of $\mathrm{Ca}^{2+} . \mathrm{Ca}^{2+}$ will stick to the troponin and cause contractions that allow us to perform voluntary activities.

\section{Myokine}

According to WHO, by 2020, chronic diseases will constitute $60 \%$ of all diseases worldwide. Center for Disease Control and Prevention suggests physical inactivity is a major cause of chronic diseases, such as diabetes mellitus, cardiovascular disease, cancer, dementia and depression. People with physical inactivity have a lifespan 5 years shorter than those with active physical activity. Type 2 diabetes mellitus, cardiovascular disease, colon cancer, breast cancer, dementia and depression are different diseases but have the same pathogenesis of physical inactivity known as diseasome of physical inactivity. ${ }^{1}$ Physical inactivity will lead to visceral fat accumulation, insulin resistance, atherosclerosis, neurodegeneration and tumors.

The striated muscle is the largest organ in the human body. The last few decades researchers have found a connection between striated muscle contractions and humoral changes known as muscle-generated exercise factor at the time of contracting and affecting metabolism in other organs such as liver and adipose tissue. Cytokines and peptides produced by striated muscles are known by the name myokine. Myokine can work in an autocrine, paracrine, or endocrine. ${ }^{4}$ Myokine gives the concept of how muscles interact with other organs. Some known myokines include IL-6, IL-8, IL-5, BDNF, FGF-21, LIF, irisin and SPARC (Figure 1).

Physical activity results in myokine secretion that can reduce inflammation due to a sedentary lifestyle. Inflammation can lead to deterioration of sarcopenia and fat accumulation in striated muscles to form a vicious circle that 


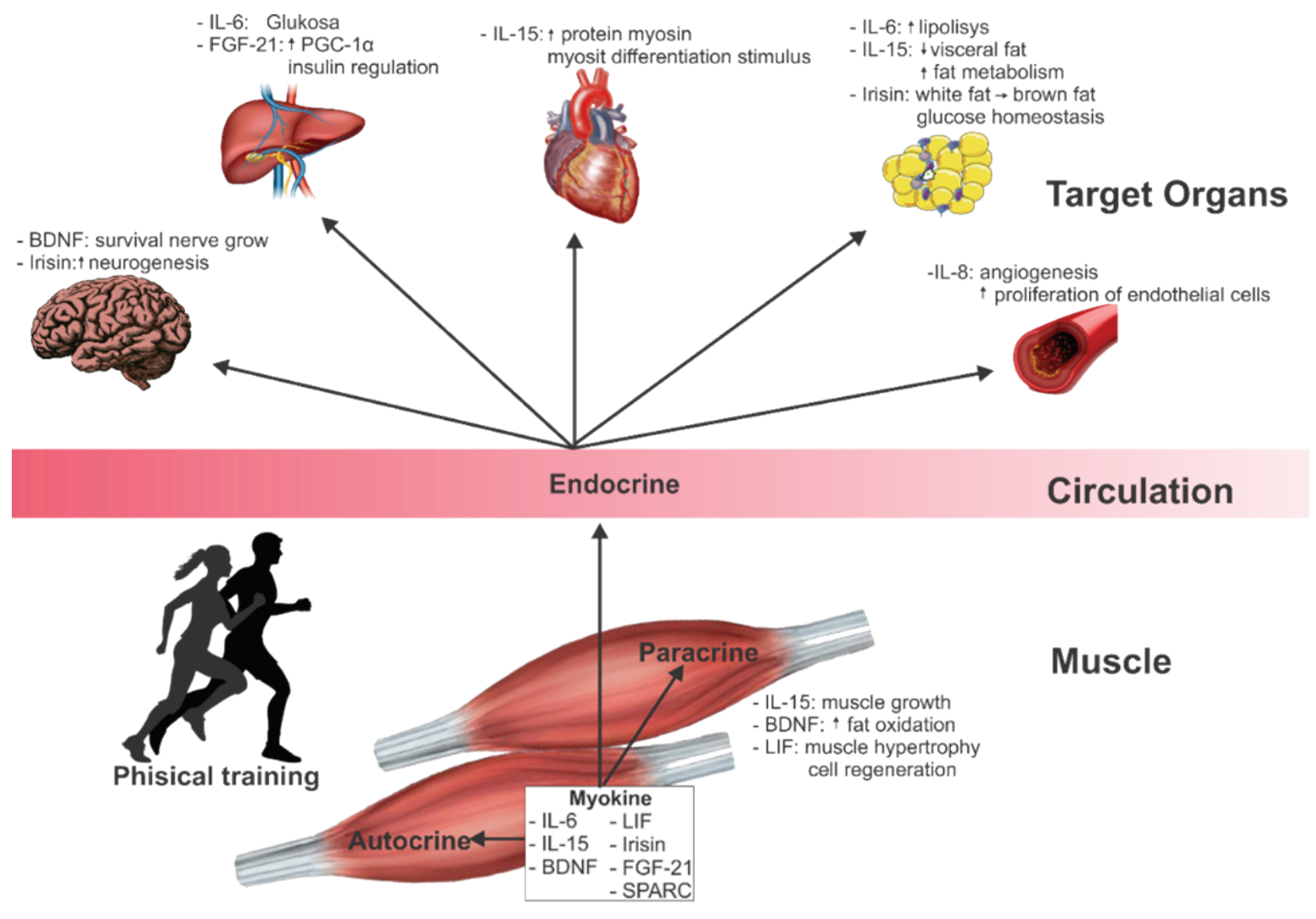

Figure 1. Myokine effect on organs. IL: Interleukin; BDNF: Brain-derived neurotrophic factor; FGF-21: Fibroblast growth factor 21; LIF: Leukemia Inhibitory Factor; PGC-1 $\alpha$ : proliferator-activated receptor $1 \alpha$. $^{1,10,14-16,20,22,23,26,28}$

will reduce muscle strength and causes physical inactivity. In addition, myokine also works racially through signals in the muscles resulting in fat oxidation. ${ }^{4-6}$ Some myokine that has been found to date will be discussed below.

\section{IL-6}

IL-6 is the first cytokine introduced as myokine. ${ }^{7}$ In 2000 it was found that IL-6 plasma increased with physical exercise. ${ }^{8}$ Afterwards, the study found that the production of IL-6 during physical activity increased especially in low muscle glycogen conditions. ${ }^{7}$ IL- 6 is produced by fiber types I and II in response to muscle contraction. ${ }^{9}$ In striated muscle IL-6 will activate AMP-activated protein kinase (AMPK) and or phosphoinositide 3 (PI3) kinase which will increase glucose uptake. In addition, IL-6 will also increase the translocation of glucose transporter type 4 (GLUT-4) from intracellular to cell plasma membrane so as to increase glucose absorption in striated muscle. This suggests an important role of myokine and striated muscle in maintaining glucose homeostasis. ${ }^{10}$
IL-6 also increases the oxidation of fatty acids in striated and whole body cells. It is mediated by AMPK by increasing the amount of cAMP and increasing the ratio of AMP : ATP. ${ }^{11}$ In addition, IL-6 in the circulation will increase glucose production by the liver during physical activity and increase lipolysis. So that the local and systemic effects of IL-6 synergistically increase the energy availability for muscle contraction. ${ }^{12}$

IL-6 was previously thought to be a proinflammatory cytokine produced by monocytes and macrophages as a response to infection stimulation. However, IL-6 produced by striated muscle through contractions does not involve other inflammatory mediators such as IL-10 and tumor necrosis factor (TNF)- $\alpha$ shows cytokine production because physical activity is different from inflammation. The IL-6 produced by macrophages must pass through the K- $\beta$ nuclear factor pathway to produce $B$ cells (NFKB) while the muscle-derived IL- 6 is activated through the $\mathrm{Ca}^{2+} /$ nuclear factor of activated T-cell (NFAT) and glycogen / p38 mitogen activated protein kinase (MAPK). When IL-6 
is produced by macrophages it produces inflammation, whereas IL-6 produced striated lymph cells do not activate an inflammatory pathway. Thus IL-6 can be an inflammatory and anti-inflammatory cytokine depending on the originator. ${ }^{13}$ During physical activity, IL-6 has an anti-inflammatory effect that suppresses the synthesis of proinflammatory cytokines such as IL- $1 \beta$ and TNF- $\alpha$ and stimulates the production of anti-inflammatory cytokines and cytokine inhibitors such as IL-1ra and IL-10.(7) A hypothesis that physical activity can increase IL-6 can regulate the metabolism of glucose and fat, so it can explain the sedentary lifestyle relationship with diseases related to glucose and fat metabolism. ${ }^{1}$

\section{IL-15}

IL-15 has anabolic effects that can increase muscle growth. IL-15 can stimulate the differentiation of myocytes and increase the amount of protein in myocytes. ${ }^{14}$ In addition, IL-15 is also thought to affect fat metabolism. There was an inverse correlation between plasma levels of IL-15 and body fat mass and elevated IL-15 levels showed a decrease in visceral fat in experimental mice. ${ }^{15}$

\section{$B D N F$}

BDNF is a neurotrophin, which is structurally similar to a growth factor that affects survival, neuronal growth so as to relate to learning and memory. BDNF works through the Trk receptor on tyrosine kinase. BDNF is mostly produced by the brain and non-neurogenic tissues including striated muscles. Alzheimer's patients have low plasma BDNF levels. In addition, low levels of BDNF are also found in patients with acute coronary syndrome and diabetes mellitus. Unlike other circulating myokines circulating, BDNF works directly on striated muscles by increasing the oxidation of fat through the AMPK pathway thus increasing adipose fat tissue. ${ }^{16}$ From this, it can be concluded that physical activity will increase fat metabolism, decreased visceral fat, decreased cardiovascular risk, diabetes mellitus, dementia through BDNF production. ${ }^{17}$

\section{IL-8}

IL-8 is a chemokine to attract neutrophils. IL- 8 increases in physical activity such as running that involve the contraction of eccentric muscles. ${ }^{18}$ The measurements of IL-8 arterial and venous concentrations showed an increase while IL-8 concentrations did not increase the systemic concentration of IL-8. This indicates that IL-8 works in an autocrine and paracrine. ${ }^{19}$ In addition, the increase in IL-8 in eccentric muscle cells did not show an increase in neutrophils and macrophages in muscle cells. The IL-8 angiogenesis effect was obtained through the activation of $\mathrm{C}-\mathrm{X}-\mathrm{C}$ chemokine receptor type 2 (CXCR2) receptor. The CXCR2 receptor increases in striated muscle biopsy after eccentric contraction. IL-8 induces angiogenesis and increases proliferation in endothelial cells. ${ }^{20}$

\section{FGF-21}

FGF-21 is known as the hormone produced by the liver during fasting. This hormone induces the liver to produce peroxisome proliferator-activated receptor $1 \alpha$ (PGC-1 $\alpha$ ) that regulates energy homeostasis, increases fatty acid oxidation, and gluconeogenesis. PGC- $1 \alpha$-deficient rats have impaired gluconeogenesis and ketogenesis. ${ }^{21}$ In experiments with infusion of insulin in healthy men, FGF21 was almost undetectable in patients prior to insulin infusion. However, after 3-4 hours there was an increase in FGF21 produced by striated muscle followed by an increase in plasma FGF-21 thus indicating FGF-21 is a myokine that serves to regulate insulin. ${ }^{22}$

\section{LIF}

LIF is a newly discovered myokine and plays an important role in muscle hypertrophy as it increases cell proliferation via the Janus kinase (JAK) pathway, transducer and activator of transcription-3 (STAT-3) signals, and phosphoinositide-3kinase. LIF affects cell proliferation so it plays an important role in hypertrophy and cell regeneration. ${ }^{23}$ LIF mRNA was found to increase after an ergometer cycle and endurance exercise. ${ }^{24,25}$ LIF has the potential to treat diseases of striated muscles such as muscular dystrophy because it increases myoblast survival in dystrophic muscle. ${ }^{24}$ However, further research is needed to find out the duet increase in LIF mRNA, the type of exercise suggested, and the time it takes to improve LIF.

\section{Irisin}

Irisin is a myokine whose production depends on the peroxisome proliferator-activated receptor- $\gamma$ coactivator-1 $\alpha$ (PPAR- $\gamma$ ) coactivator- $1 \alpha$ (PGC1- $\alpha$ ), where PGC1- $\alpha$ will stimulate the expression of fibronectin gene type III domaincontaining protein 5 (FNDC5) which encodes irisin. Irisin works on adipose tissue by inducing the conversion of white fat to brown fat. This increases the increase in energy requirements thus improving glucose homeostasis. In 
addition, FNDC5 is also expressed in the brain. Increased irisin will increase FNDC5 in the brain which will increase BDNF resulting in neurogenesis in the brain. The FNDC5/ irisin pathway is a pathway that explains the relationship between physical exercise and the brain. ${ }^{26}$ In addition, irisin also regulates muscle metabolism. Muscles given in vitro irisin will increase the expression of PGC- $1 \alpha$, mitochondrial transcription factor A (TFAM), GLUT4 and will strengthen the signal between PGC- $1 \alpha$ and FNDC5 resulting in increased energy demand and metabolism in the muscle.

\section{SPARC}

The SPARC, also known as B40, was first discovered on the bone and was named osteonectin. But recent studies have also found that SPARC is found in myoblasts, myotubes, and muscle fibers. SPARC is one of myokine that plays a role in fat accumulation and glucose metabolism. SPARC modulates fibronectin which is the inhibitor of extracellular matrix production during adipogenesis. This interruption of extracellular matrix production results in fiber regulatory constraints for preadipocyte cell structures. ${ }^{27}$ SPARC also plays a role in regulating blood sugar through AMPK signals that can stimulate glucose uptake by cells through enhancement of GLUT translocation. ${ }^{28}$

\section{The Release of Myokine by Contracting Muscles}

The role of cytokines in the pathophysiology of the disease is growing rapidly. It has been known for a long time that the concentration of IL-6 increases during physical activity but the origin of this increase is only known since 2000 . IL-6 is produced by skeletal muscle and is released into circulation so that IL-6 is one of the cytokines released by striated muscle, which known as myokine. ${ }^{29}$ The concentration of IL-6 in plasma increases up to 100 -fold during physical exercise. This increase is also accompanied by an increase in the inhibitor of the antagonist interleukine-1 receptor cytokine (IL-1ra) and anti-inflammatory cytokines such as IL-10. Initially, estimated IL-6 increase after physical activity is caused by an immunological response caused by local damage from striated muscle after physical exercise and immune cells. However, the results of the cytometric examination showed a decrease in the number, percentage, and mean of monocyte intensity which gave positive replication to IL-6 after physical exercise, so it can be concluded that IL-6 increased after physical activity did not involve the immune system. ${ }^{12}$
The striated muscle in humans is a unique organ that can produce IL-6 during contractions without an increase in inflammatory markers. The expression of IL-6 mRNA is affected by muscle glycogen levels where there is an increase in IL-6 mRNA levels in muscles with low glycogen. There are several hypotheses about the way in which IL-6 gene transcription is stimulated. At a time when the carbohydrates in the muscles decrease, a sympathoadrenal response occurs which results in epinephrine expulsion that stimulates the transcription of IL- 6 gene through $\beta$-adrenergic stimulation of protein kinase A. However, after experiments using rats it is found that the increase in m-RNA IL-6 occurs only on administration of epinephrine a dose of $1000 \mathrm{nmol}$, but not on physiological dosage (10 and $100 \mathrm{nmol})$. In addition, transcription of the IL- 6 gene is also estimated through the release of calcium $\left(\mathrm{Ca}^{2+}\right)$ in the lateral pouch of the endoplasmic reticulum. The striated muscle gave calcium ionophore ionomycin $5 \mathrm{nmol}$ will increase the expression of IL-6 mRNA. ${ }^{30}$

In the contraction experiment, we measured the difference between IL- 6 artery and vein on contracting and non-contraction muscles. The difference in contraction of IL-6 was found to be significantly greater. ${ }^{8}$ In addition, other studies have also shown that there is an increased concentration of IL-6 in the biopsy of ribonucleic acid (mRNA) muscle contractors. ${ }^{12}$ It can therefore be concluded that the transcription of IL- 6 gene during contraction is determined by the levels of calcium and glycogen levels.

To investigate intracellular signals that play a role in transcription of IL- 6 gene, experiments were conducted in two circumstances with normal and low carbohydrate diets and biopsies were performed to determine levels of IL-6 mRNA and nuclear proteins such as c-Jun N-terminal kinases (JNK) and MAPK. There was an increase in IL-6 in the contracting muscle. There was also an increase in phosphorylation of p38 in muscles previously given a lowcarbohydrate diet. ${ }^{31}$

\section{Sarcopenia}

Sarcopenia is the term first introduced by Irwin Rosenberg in 1989. Sarcopenia comes from the Greek word "Sarco" which means meat or muscle and "penia" which means deficiency. Sarcopenia is defined as a lack of muscle mass. Currently, the term is aimed at a loss of muscle mass and strength with age. ${ }^{32}$ Loss of muscle mass was first observed by an English neurologist named MacDonald Critchley who 
discovered the loss of muscle mass in the hands and feet of geriatric patients. Currently, sarcopenia is defined as loss of muscle mass in the elderly below 2 standard deviations of the average young adult muscle mass. ${ }^{33}$

Based on the epidemiological data the prevalence of sarcopenia is $30 \%$ at the age of more than 60 years, and $50 \%$ at the age of more than 80 years. ${ }^{33}$ It is estimated there are 3.6 million people in the United States who have sarcopenia. Sarcopenia is a complex medical condition characterized by progressive loss of muscle mass, the quality and function of skeletal muscles associated with aging. Sarcopenia also causes a decrease in muscle strength. A decrease in muscle strength is one of the causes of frailty. Frailty can cause a person to be vulnerable to stress were experiencing a little stress will cause their condition to deteriorate like hospitalization, disability, or even death. ${ }^{2}$ Symptoms of frailty are a weakness, inability to climb one ladder, inability to walk a block, have more than five diseases, and weight loss. ${ }^{33}$

There are various risk factors for the occurrence of sarcopenia that can be divided into some factors.

\section{Constitutional factors}

Age and sex affect sarcopenia. Low birth weight increases the risk of sarcopenia.

\section{Aging factor}

Aging causes increased catabolic stimulation and decrease anabolic stimulation. Hormonal dysregulation such as testosterone, growth hormone and insulin like growth factor (IGF)-1 also occur during aging. Muscle contractions resulting from physical exercise will lead to the release of growth factors such as IGF-Ea and cause protein synthesis and muscle regeneration. In aging physical inactivity often occurs so that the release of IGF becomes reduced and leads to decreased muscle synthesis. Testosterone also plays a role in decreasing muscle mass and strength. Testosterone decreases by $1 \%$ after the age of 30 years in men while starting to decline at the age of 25 to 40 years in women. ${ }^{34}$ Several studies have shown that low doses of testosterone can increase muscle mass. ${ }^{35}$ While in other studies showed the provision of testosterone in frailty patients associated with decreased mortality compared with placebo. ${ }^{36}$ In adults, growth hormone increases nitrogen retention and muscle mass. Vitamin D $25(\mathrm{OH})$ will decrease with aging. Vitamin D $25(\mathrm{OH})$ levels in old age will cause sarcopenia, fall, hip fracture and death. ${ }^{36}$

\section{Lifestyle}

Decreased intake of foods, especially protein with physical activity that less increases the risk of sarcopenia. Nutrition is needed to maintain muscle mass. Geriatric patients require 1.2 grams of protein/kilogram of body weight per day. Amino leucine acids can increase protein synthesis better than other amino acids. Leucine-containing foods include milk, cheese, beef, tuna, chicken, beans, soybeans and eggs. ${ }^{37}$ Consumption of alcohol and cigarettes also increases the risk of sarcopenia.

\section{Changes in body condition}

Late bed rest, immobilization, and less weight increase the risk of sarcopenia.

\section{Chronic illness}

Chronic diseases such as diabetes, advanced functional organ failure, cognitive disorders and mood disorders cause chronic inflammation that can lead to sarcopenia.

\section{Sarcopenia and Aging}

Sarcopenia occurs due to the multifactorial process of losing alpha motor nerve inputs to the spinal columns, physical activity, hormonal changes, energy, protein intake, oxidative stress and inflammatory processes. ${ }^{38}$ Sarcopenia due to aging is explained by the immunosenescence that is the decrease of the immune system, resulting in low-grade inflammatory conditions in the long term is often called chronic inflammation aging conditions (Figure 2). This inflammatory process is based on $\mathrm{CD} 68^{+}$and macrophage infiltration resulting in anabolic and catabolic protein imbalance. In the aging process occurs mitochondrial dysfunction as an energy producer, redox regulation, and communication with cell nuclei, gene expression, and

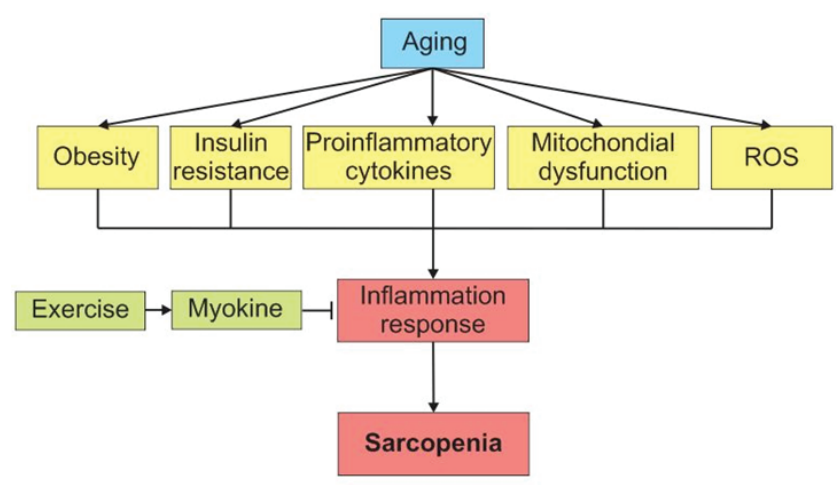

Figure 2. The relationship of aging with sarcopenia. ${ }^{39}$ 
apoptotic processes. So mitochondria function to maintain their functional integrity and structure during the process of biogenesis, antioxidants, fusion and fission dynamics, and autophagy. PGC- $1 \alpha$ functions to control transcriptional regulation of some nuclei and mitochondrial genes. So PGC$1 \alpha$ can play a role in controlling the function and muscle mass. Activation PGC-1 $\alpha$ protects muscle from degradation and destruction by proteolysis, oxidative, inflammation and aproposis. Physical exercise is a very powerful stimulation stimulating PGC- $1 \alpha$ expression. ${ }^{39}$

Chronic inflammation that produces many proinflammatory cytokines will activate signal catabolism and upregulation of inflammatory pathways such as NF-KB and STAT3 thereby increasing the activation of ubiquitinproteasome and autophagy systems. This process not only increases proinflammatory cytokines but also inhibits the secretion of anti-inflammatory cytokines such as IL-10. Cytokines are produced by various inflammatory cells and stoma cells as well as skeletal muscle cells. In mature myofiber muscle cells will form muscle mass while myotine will express m RNA of various cytokines. ${ }^{39}$ Several factors can reduce and even prevent inflammation that causes sarcoopenia, such as exercise, nutritional intake, hormonal therapy and pharmacotherapy.

Enforcement of sarcopenia diagnosis is based on various risk factors reinforced by muscle weakness, fatigue, low endurance associated with a decreased speed of walking, movement disorders and inability to perform daily tasks. Enforcement of sarcopenia diagnosis is based on various risk factors reinforced by muscle weakness, fatigue, low endurance associated with a decreased speed of walking, movement disorders and inability to perform daily tasks. Based on the European Working Group on Sarcopenia in Older People (EWGSOP), a criterion for sarcopenia is the loss of muscle mass coupled with one of two conditions: loss of muscle strength and or loss of performance. ${ }^{40,41}$ Physical performance can be measured with gait speed, up stair test and time up and go test. If one of the tests gives a positive result of walking speed $<0.8$ meters/second and can not stand from the chair then the muscle mass measurements are taken. Examination of muscle mass using bioelectrical impedance (BIA) by measuring the ratio between muscle mass and fat mass. If still found the results are dubious then conducted a dual energy inspection X-ray absorptiometry (DEXA). While the gold standard to determine the ratio of muscle mass and fat mass is through computed tomography (CT) scanning or magnetic resonance imaging (MRI).
Muscle strength can be measured by 2 way tests, which are grasping strength tests and knee extension tests. The criterion for decreasing muscle strength according to AWGS is less than 20 percentile of the average population of grasping strength tests (Figure 3 ). ${ }^{42}$

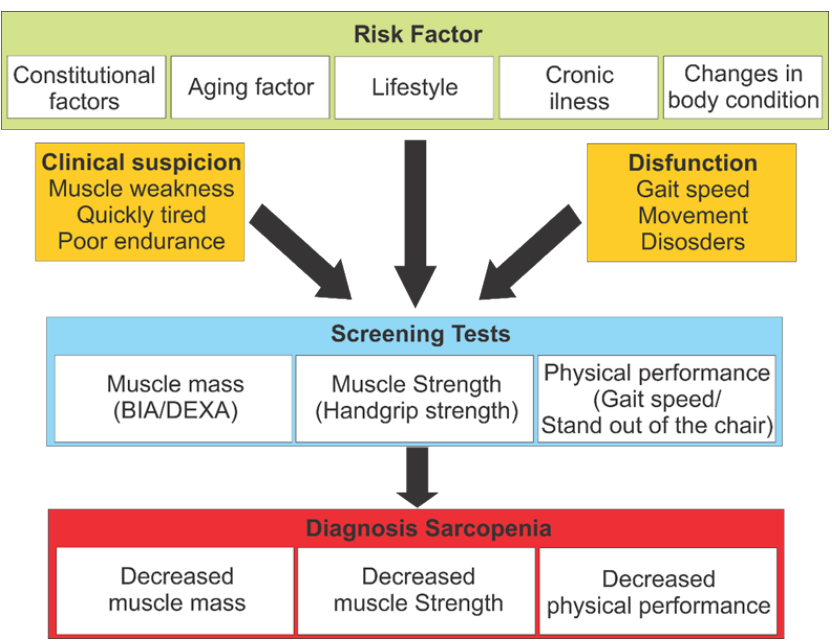

Figure 3. Clinical approach of sarcopenia diagnosis. BIA: bioelectrical impedance (BIA); DEXA: dual energy X-ray absorptiometry. ${ }^{42}$

\section{The Role of Physical Exercise in Myokine Enhancement}

Physical exercise can induce myokine secretion from striated muscle to circulation. In conditions of metabolic climates such as diabetes mellitus and obesity, adipose tissue will amplify proinflammatory cytokines that cause pathological processes such as atherosclerosis and insulin resistance. In addition, myokine expenditure can inhibit the effects of adipokines. In response to exercise, striated muscles release myokines such as irisin, IL-15, LIF, BDNF, FGF-21 and SPARC that have local effects on muscles or to target organs.

Table 1 shows the studies that is showing the relationship between physical exercise and increased serum myokine.

\section{The Role of Physical Exercise in Chronic Inflammation in Geriatric Patients}

Chronic inflammation of geriatric populations causes a loss of muscle mass resulting in disability and even death. The levels of pro-inflammatory cytokines in the geriatric population were higher than in the young adult population. 
Table 1. Research on physical exercise that induces myokine. ${ }^{43}$

\begin{tabular}{|c|c|c|}
\hline Myokine & Physical training & Results \\
\hline IL-6 & Exercise knee extensor contraction (concentric) for 5 hours & $\uparrow \mathrm{IL}-6(8)$ \\
\hline \multirow[t]{6}{*}{$\overline{\mathrm{IL}}-15$} & Treadmill & $\uparrow$ serum IL-15 (44) \\
\hline & ( $70 \%$ maximum heart rate, 30 minutes) & \\
\hline & Resistance training & $\uparrow$ IL-6, IL-10, IL-1ra, IL-8 (45) \\
\hline & ( 4 sets, 10 repetitions, $2-3$ minutes break every interval) & \\
\hline & Resistance training & $\uparrow$ serum IL- 15 (46) \\
\hline & (3 days per week, $6-10$ repetitions) & \\
\hline \multirow[t]{2}{*}{$\overline{\mathrm{BDNF}}$} & Cycling & 个BDNF MRNA, \\
\hline & (VO2 maximum of $60 \%, 120$ minutes) & $\uparrow$ protein BDNF (16) \\
\hline \multirow[t]{3}{*}{$\overline{\mathrm{LIF}}$} & Resistance training & $\uparrow$ LIF MRNA up to 9 times (24) \\
\hline & Cycle ergometer & $\uparrow$ LIF MRNA up to 4.5 times (25) \\
\hline & ( 3 hours, VO2 maximum $60 \%$ ) & \\
\hline \multirow[t]{3}{*}{ Irisin } & Endurance exercise & $\uparrow$ plasma irisin \\
\hline & (cycling, maximum VO2 65\%, 4-5 sessions) & \\
\hline & Acute exercise & $\uparrow$ plasma irisin (47) \\
\hline \multirow[t]{2}{*}{ FGF-21 } & Treadmill & $\uparrow \mathrm{FGF}-21(48)$ \\
\hline & (Bruce's protocol 5 days/2 weeks, 21 minutes) & \\
\hline \multirow[t]{2}{*}{ SPARC } & Acute exercise & $\uparrow$ SPARC (49) \\
\hline & (cycling, VO2 maximum $70 \%, 30$ minutes) & \\
\hline
\end{tabular}

BDNF: brain-derived neurotropic factor; FGF-21: fibroblast growth factor 21; IL: interleukin; LIF: leukemia inhibitory factor; SPARC: secreted protein acidic and rich in cysteine.

Some cytokines have a poor direct effect on striated muscle causing loss of muscle fibers, so the inflammatory pathway is a target of intervention that can decrease the incidence of disability in the geriatric population. The use of antiinflammatory can indeed reduce inflammation that occurs in geriatric patients, but the side effects caused a limitation of the use of an anti-inflammatory. To date, no pharmacological drugs with anti-inflammatory effects have been used to treat sarcopenia in geriatric populations. Regular physical exercise is the only therapy to improve physical function in the geriatric population.

Large observational studies such as the British Regional Heart Study, the Third National Health and Nutrition Examination Survey (NHANESIII), and the Cardiovascular Health Study (CHS), show an inverse relationship between CRP concentration and physical activity in the elderly. In the ABC Health study also found a linear decrease in TNF tendency with increased physical activity. ${ }^{5}$ In NHANESIII study, physical activity of more than 22 times per month decreased CRP by $63 \%$ compared with physical activity less than 3 times per month without being affected by BMI and waist to hip ratio. ${ }^{1}$ Other studies also showed aerobic exercise decreased the production of atherogenic cytokines by mononuclear cells such as interferon $\gamma$, TNF $\alpha$ and IL-1 and increased production of atheroprotective cytokines such as IL-10, IL-4 and transforming growth factor (TGF)- $\beta 1$ increased by $36 \%{ }^{28}$ The cause of this is increased myokine during physical exercise. As mentioned before, the striated muscle that expresses myokine, especially IL-6 which will cause metabolic changes during physical activity. The release of IL-6 to more than 100-fold during contractions in addition to enhancing anti-inflammatory cytokines such as IL-1ra and IL-10 may also decrease the production of TNF $\alpha$ and IL- $1 \beta{ }^{16}$

\section{Conclusion}

Chronic inflammation is a contributor that plays a role in the pathophysiology of various diseases including sarcopenia. Provision of therapy to reduce inflammation is crucial, but the pharmacological approach has not shown significant results. Various studies have shown that physical activity can stimulate myokine secretion from contracted striated muscles. Some known myokines include IL-6, IL-8, IL- 
5, BDNF, FGF-21, LIF, irisin and SPARC. Myokine can affect the metabolism of glucose, fatty acids, angiogenesis, neurogenesis and can explain the relationship between striated muscles with the liver, fat tissue and brain. Through this mechanism, physical activity is expected to reduce the incidence of sarcopenia and diseasome of physical inactivity, especially in geriatric populations.

\section{References}

1. Pedersen BK. The Diseasome of Physical Inactivity - and the Role of Myokines in Muscle-fat Cross Talk. J Physiol. 2009; 587(3): 555968.

2. Morley JE. Sarcopenia in the Elderly. Fam Pract. 2012; 29 (Suppl 1): i44-8.

3. Pratesi A, Tarantini F, Bari M Di. Skeletal Muscle: An Endocrine Organ. Clin Cases Miner Bone Metab. 2013; 10(1): 11-4.

4. Pedersen BK. Muscles and Their Myokines. J Exp Biol. 2011; 214(2): 337-46.

5. Brandt C, Pedersen BK. The Role of Exercise-Induced Myokines in Muscle Homeostasis and the Defense against Chronic Diseases. J Biomed Biotechnol. 2010; 2010: 1-6.

6. Petersen AMW, Pedersen BK. The Anti-inflammatory Effect of Exercise. J Appl Physiol. 2005; 98(4): 1154-62.

7. Steensberg A, Fischer CP, Keller C, Møller K, Pedersen BK. IL-6 Enhances Plasma IL-1ra, IL-10, and Cortisol in Humans. Am J Physiol - Endocrinol Metab. 2003; 285(2): E433-7.

8. Steensberg A, Van Hall G, Osada T, Sacchetti M, Saltin B, Pedersen BK. Production of Interleukin-6 in Contracting Human Skeletal Muscles Can Account for the Exercise-induced Increase in Plasma Interleukin-6. J Physiol. 2000; 529(1): 237-42.

9. Hiscock N. Skeletal Myocytes are a Source of Interleukin-6 mRNA Expression and Protein Release During Contraction: Evidence of Fiber Type Specificity. FASEB. 2004; 18(9): 992-4.

10. Carey AL, Steinberg GR, Macaulay SL, Thomas WG, Holmes AG, Ramm G, et al. Interleukin-6 Increases Insulin-stimulated Glucose Disposal in Humans and Glucose Uptake and Fatty Acid Oxidation in vitro via AMP-activated Protein Kinase. Diabetes. 2006; 55(10): 2688-97.

11. Ruderman NB, Keller C, Richard AM, Saha AK, Luo Z, Xiang X, et al. Interleukin-6 Regulation of AMP-activated Protein Kinase: Potential Role in The Systemic Response to Exercise and Prevention of The Metabolic Syndrome. Diabetes. 2006; 55 (Suppl 2): S4854.

12. Febbraio Ma. Muscle-derived Interleukin-6: Mechanisms for Activation and Possible Biological Roles. FASEB J. 2002; 16(11): 1335-47.

13. Pedersen BK, Febbraio MA. Muscle as an Endocrine Organ: Focus on Muscle-Derived Interleukin-6. Physiol Rev. 2008; 88(4):1379406.

14. Quinn LS, Haugk KL, Grabstein KH. Interleukin-15: A Novel Anabolic Cytokine for Skeletal Muscle. Endocrinol. 1995; 136(8): 3669-72.

15. Nielsen AR, Hojman P, Erikstrup C, Fischer CP, Plomgaard P, Mounier R, et al. Association between Interleukin-15 and Obesity: Interleukin-15 as a Potential Regulator of Fat Mass. J Clin Endocrinol Metab. 2008; 93(11): 4486-93.
16. Matthews VB, Åström MB, Chan MHS, Bruce CR, Krabbe KS, Prelovsek $\mathrm{O}$, et al. Brain-derived Neurotrophic Factor is Produced by Skeletal Muscle Cells in Response to Contraction and Enhances Fat Oxidation via Activation of AMP-activated Protein Kinase. Diabetologia. 2009; 52(7): 1409-18.

17. Whitmer RA, Gustafson DR, Barrett-Connor E, Haan MN, Gunderson EP, Yaffe K. Central Obesity and Increased Risk of Dementia More than Three Decades Later. Neurology. 2008; 71(14): 1057-64.

18. Nielsen AR, Pedersen BK. The Biological Roles of Exercise-induced Cytokines: IL-6 , IL-8 , and IL-15. Appl Physiol Nutr Metab. 2007; 32(5): 833-9.

19. Akerstrom T, Steensberg A, Keller P, Keller C, Penkowa M, Pedersen BK. Exercise Induces Interleukin-8 Expression in Human Skeletal Muscle. 2005; 563(2): 507-16.

20. Frydelund-Larsen L, Penkowa M, Akerstrom T, Zankari A, Nielsen S, Pedersen BK. Exercise Induces Interleukin-8 Receptor (CXCR2) Expression in Human Skeletal Muscle. Exp Physiol. 2007; 92(1): 233-40.

21. Potthoff MJ, Inagaki T, Satapati S, Ding X, He T, Goetz R, et al. FGF21 Induces PGC-1 and Regulates Carbohydrate and Fatty Acid Metabolism During The Adaptive Starvation Response. Proc Natl Acad Sci. 2009; 106(26): 10853-8.

22. Hojman P, Pedersen M, Nielsen AR, Krogh-Madsen R, Yfanti C, Åkerstrom T, et al. Fibroblast Growth Factor-21 is Induced in Human Skeletal Muscles by Hyperinsulinemia. Diabetes. 2009; 58(12): 2797-801.

23. Broholm C, Pedersen BK. Leukaemia Inhibitory Factor - An ExerciseInduced Myokine. Exerc Immunol Rev. 2010; 16: 77-85.

24. Hunt LC, Anthea Coles C, Gorman CM, Tudor EM, Smythe GM, White JD. Alterations in The Expression of Leukemia Inhibitory Factor Following Exercise: Comparisons between Wild-type and mdx Muscles. PLoS Curr. 2011; 3: 1277. doi:10.1371/currents. RRN1277.

25. Broholm C, Mortensen OH, Nielsen S, Akerstrom T, Zankari A, Dahl $\mathrm{B}$, et al. Exercise Induces Expression of Leukaemia Inhibitory Factor in Human Skeletal Muscle. J Physiol. 2008; 586(8): $2195-$ 201.

26. Wrann CD, White JP, Salogiannnis J, Laznik-Bogoslavski D, Wu J, Ma D, et al. Exercise Induces Hippocampal BDNF through a PGC1 $\alpha /$ FNDC5 Pathway. Cell Metab. 2013; 18(5): 649-59.

27. Nie J, Helene Sage E. SPARC Inhibits Adipogenesis by Its Enhancement of $\beta$-catenin signaling. J Biol Chem. 2009; 284(2): 1279-90.

28. Fryer LGD, Foufelle F, Barnes K, Baldwin SA, Woods A, Carling D. Characterization of The Role of The AMP-activated Protein Kinase in The Stimulation of Glucose Transport in Skeletal Muscle Cells. Biochem J. 2002; 363(1): 167-74.

29. Febbraio MA, Pedersen BK. Contraction-induced Myokine Production and Release: Is Skeletal Muscle An Endocrine Organ? Exerc Sport Sci Rev. 2005; 33(3): 114-9.

30. Holmes AG, Watt MJ, Carey AL, Febbraio MA. Ionomycin, but Not Physiologic Doses of Epinephrine, Stimulates Skeletal Muscle Interleukin-6 mRNA Expression and Protein Release. Metabolism. 2004; 53(11): 1492-5.

31. Chan Mhs, Mcgee Sl, Watt Mj, Hargreaves M, Febbraio Ma. Altering Dietary Nutrient Intake That Reduces Glycogen Content Leads to Phosphorylation of Nuclear p38 MAP Kinase in Human Skeletal Muscle: Association with IL-6 Gene Transcription During Contraction. FASEB J. 2004;18(14): 1785-7. 
32. Rosenberg IH. Epidemiologic and methodologic problems in determining nutritional status of older persons. Am J Clin Nutr. 1989; 50 (Suppl 5): 1121-235.

33. Van Kan G A, YM R, JE M, B V. Frailty: Toward A Clinical Definition. J Am Med Dir Assoc. 2008; 9: 71-2.

34. Perry HM, Miller DK, Patrick P, Morley JE. Testosterone and Leptin in Older African-American Men: Relationship to Age, Strength, Function, and Season. Metabolism. 2000; 49(8): 1085-91.

35. Wang C, Nieschlag E, Swerdloff R, Behre HM, Hellstrom WJ, Gooren LJ, et al. Investigation, Treatment and Monitoring of Lateonset Hypogonadism in Males. Eur J Endocrinol. 2008; 159(5): 507-14.

36. Morley JE. Vitamin D Redux. J Am Med Dir Assoc. 2009; 10(9): 591-2.

37. Millward DJ, Fereday A, Gibson NR, Cox MC, Pacy PJ. Efficiency of Utilization of Wheat and Milk Protein in Healthy Adults and Apparent Lysine Requirements Determined by A Single-meal [113C] Leucine Balance Protocol. Am J Clin Nutr. 2002; 76(6): 132634.

38. Setiati S, Rizka A. Sarkopenia. In: Buku Ajar Ilmu Penyakit Dalam. III. Jakarta: Balai Penerbit FKUI; 2004. p. 3717-24.

39. Fan J, Kou X, Yang Y, Chen N. MicroRNA-regulated Proinflammatory Cytokines in Sarcopenia. Mediators Inflamm. 2016; 2016: 1438686, doi: 10.1155/2016/1438686.

40. Jentoft AJC, Baeyens JP, Bauer JM, Boirie Y, Cederholm T, Landi F, et al. Sarcopenia: European Consensus on Definition and Diagnosis. Age Ageing. 2010; 39(4): 412-23.

41. Giannoulis MG, Martin FC, Nair KS, Umpleby AM, Sonksen P. Hormone Replacement Therapy and Physical function in healthy older men. Time to talk hormones? Endocr Rev. 2012; 33(3): 31477
42. Rodríguez-Mañas L, Féart C, Mann G, Viña J, Chatterji S, ChodzkoZajko W, et al. Searching for An Operational Definition of Frailty: A Delphi Method-based Consensus Statement. The Frailty Operative Definition-consensus Conference Project. J Gerontol Ser A Biol Sci Med Sci. 2013; 68(1): 62-7.

43. So B, Kim H, Kim J, Song W. Exercise-induced Myokines in Health and Metabolic Diseases. Integr Med Res. 2014; 3(4): 172-9.

44. Tamura Y, Watanabe K, Kantani T, Hayashi J, Ishida N, Kaneki M. Upregulation of Circulating IL-15 by Treadmill Running in Healthy Individuals: Is IL-15 An Endocrine Mediator of The Beneficial Effects of Endurance Exercise? Endocr J. 2011; 58(3): 211-5.

45. Nieman DC, Davis JM, Brown VA, Henson DA, Dumke CL, Utter $\mathrm{AC}$, et al. Influence of Carbohydrate Ingestion on Immune Changes After $2 \mathrm{~h}$ of Intensive Resistance Training. J Appl Physiol. 2004; 96(4): 1292-8.

46. Riechman SE. Association of Interleukin-15 Protein and Interleukin-15 Receptor Genetic Variation with Resistance Exercise Training Responses. J Appl Physiol. 2004; 97(6): 2214-9.

47. Huh JY, Panagiotou G, Mougios V, Brinkoetter M, Vamvini MT, Schneider BE, et al. FNDC5 and Irisin in Humans: I. Predictors of Circulating Concentrations in Serum and Plasma and II. mRNA Expression and Circulating Concentrations in Response to Weight Loss and Exercise. Metabolism. 2012; 61(12): 1725-38.

48. Cuevas-Ramos D, Almeda-Valdés P, Meza-Arana CE, Brito-Córdova G, Gómez-Pérez FJ, Mehta R, et al. Exercise Increases Serum Fibroblast Growth Factor 21 (FGF21) levels. PLoS One. 2012;7(5). e38022. doi: 10.1371/journal.pone.0038022.

49. Aoi W, Naito Y, Takagi T, Tanimura Y, Takanami Y, Kawai Y, et al. A Novel Myokine, Secreted Protein Acidic and Rich in Cysteine (SPARC), Suppresses Colon Tumorigenesis via Regular Exercise. Gut. 2013; 62(6): 882-9. 